\title{
Clinical efficacy of first-generation EGFR-TKIs in patients with advanced non-small-cell lung cancer harboring EGFR exon 20 mutations
}

This article was published in the following Dove Press journal:

OncoTargets and Therapy

8 July 2016

Number of times this article has been viewed

\author{
Dan Chen' \\ Zhengbo Song ${ }^{2}$ \\ Guoping Cheng ${ }^{3}$ \\ 'Department of Cardiothoracic \\ Surgery, The First Affiliated Hospital \\ of Chongqing Medical University, \\ Chongqing, ${ }^{2}$ Department of \\ Chemotherapy, ${ }^{3}$ Department of \\ Pathology, Zhejiang Cancer Hospital, \\ Hangzhou, People's Republic of China
}

Correspondence: Guoping Cheng Department of Pathology, Zhejiang Cancer Hospital, 38 Guangji Road, 310022 Hangzhou, People's Republic of China

Tel/fax +86 57I 88I2 2082

Email zjzlyyII@I63.com

Zhengbo Song

Department of Chemotherapy, Zhejiang

Cancer Hospital, 38 Guangji Road,

310022 Hangzhou, People's Republic

of China

Tel/fax $+8657 / 88122080$

Email songzhengbo83@163.com
Purpose: Subsets of non-small-cell lung cancer patients with epidermal growth factor receptor (EGFR) mutations carry uncommon subtypes. We evaluated the efficacy of first-generation EGFR-tyrosine kinase inhibitors (TKIs; erlotinib, gefitinib, and icotinib) in patients with nonsmall-cell lung cancer carrying insertions and T790M and S768I mutations in EGFR exon 20. Patients and methods: Patients carrying EGFR exon 20 insertion/T790M/S768I mutations and treated with EGFR-TKIs were evaluated from 2005 to 2014 in Zhejiang Cancer Hospital. The efficacy was evaluated using the Kaplan-Meier method and compared with the log-rank test. Results: Sixty-two patients with exon 20 insertion/T790M/S768I mutations were enrolled. Mutations including exon 20 insertions and T790M and S768I mutations were observed in 29,23 , and ten patients, respectively. In total, the response rate and median progression-free survival (PFS) were $8.1 \%$ and 2.1 months, respectively. Patients with S768I mutation manifested the longest median PFS (2.7 months), followed by those with T790M (2.4 months) and exon 20 insertions ( 1.9 months; $P=0.022$ ). Patients with complex mutations show a better PFS than those with single mutations ( 2.7 months vs 1.9 months; $P=0.034$ ).

Conclusion: First-generation EGFR-TKIs are less effective in patients with exon 20 uncommon mutations than in those with common mutations. Patients with complex mutations benefited more from first-generation EGFR-TKIs than those with single mutations.

Keywords: non-small cell lung cancer, epidermal growth factor receptor, EGFR mutations, exon 20, tyrosine kinase inhibitor

\section{Introduction}

Lung cancer is the leading cause of cancer-related deaths in the People's Republic of China. ${ }^{1}$ Non-small-cell lung cancer (NSCLC) constitutes $\sim 70 \%-80 \%$ of all lung cancers. ${ }^{2}$ Somatic mutations within the epidermal growth factor receptor (EGFR) gene were discovered in a subset of NSCLC patients, especially in East Asian female patients with adenocarcinoma. ${ }^{3-6}$ Treatments targeting EGFR mutations incorporate EGFR inhibitors such as gefitinib and erlotinib, resulting in extended survival and increased quality of life in patients with NSCLC. ${ }^{7-10}$

Two major EGFR-activating mutations include an in-frame deletion in exon 19 (del 19) and an L858R substitution in exon 21. These mutations account for $\sim 90 \%$ of all clinically important mutations related to EGFR-tyrosine kinase inhibitor (TKI) sensitivity. ${ }^{11,12}$ In addition, other uncommon mutations have been identified. Among these, the G719X in exon 18 and L861Q in exon 21 have been frequently reported. The published data demonstrate moderate sensitivity of these two mutations to EGFR-TKI treatment, resulting in a median progression-free survival (PFS) of $\sim 6$ months. ${ }^{13-15}$ 
T790M mutation, S768I mutation, and insertions have been identified in exon 20. Owing to the rarity of these subtypes, the mutations have not been fully described. ${ }^{16,17}$ The efficacy of EGFR-TKIs in patients harboring these mutations to EGFR-TKIs is still unknown.

This study investigated the clinical characteristics and efficacy of EGFR-TKIs in patients carrying uncommon mutations in EGFR exon 20.

\section{Patients and methods}

This study conducted from 2005 to 2014 at the Zhejiang Cancer Hospital (People's Republic of China) included patients with known EGFR genotypes and treated for advanced NSCLC using EGFR-TKIs (gefitinib, erlotinib, or icotinib). The protocol was reviewed and approved by the Ethics Committee of Zhejiang Cancer Hospital. All patients provided written informed consent.

\section{EGFR mutational analysis}

The tumor EGFR mutational status was determined by analyzing the DNA extracted from formalin-fixed, paraffin-embedded tumor tissues or from cytology samples. All samples were tested using an amplification refractory mutation system-based EGFR mutation detection kit (Amoy Diagnostics, Xiamen, People's Republic of China). The method enabled the detection of 29 mutations in exons $18,19,20$, and 21 . In exon 20, two mutations (S768I and T790M) and one insertion were included.

\section{Efficacy evaluation}

Tumor responses were assessed based on the Response Evaluation Criteria in Solid Tumors Version 1.1. Objective tumor responses included complete response (CR), partial response (PR), stable disease (SD), and progressive disease. Objective responses included the CR and PR. Disease control rate was defined as the addition of objective response and stabilization rates $(\mathrm{CR}+\mathrm{PR}+\mathrm{SD})$.

\section{Statistical analysis}

PFS and overall survival (OS) were estimated using the KaplanMeier method. The log-rank test was used to compare the PFS and OS in different patients. PFS was calculated from the starting date of EGFR-TKIs treatment until the date of disease progression or death. The OS was measured from the date of initial dosing of EGFR-TKIs until the date of the last follow-up or death. All statistical analyses were performed using Statistics 17.0 (SPSS Inc., Chicago, IL, USA). The median follow-up time was 18.5 months (range 5.0-36 months), and the last follow-up date was August 1, 2015.

\section{Results \\ Patient profile}

Between January 2005 and July 2014, 3,910 patients were evaluated for EGFR mutations. The results showed that 2,350 patients $(60.1 \%)$ carried wild-type EGFR and 1,560 patients harbored EGFR mutations. Eighty-nine patients carried exon 20 mutations, including 62 treated with EGFR-TKIs. The clinical profile of patients is listed in Table 1. Patients included 39 males and 23 females with a median age of 60 years. Thirty-one patients were smokers or ever-smokers. The performance status of $0-1$ was observed in $82.3 \%$ patients, while a performance status of 2 or 3 was seen in eleven patients. Histologically, adenocarcinoma was the most common subtype (56 cases, 90.3\%), and adenosquamous or large cell carcinoma occurred in six patients. At the start of EGFR-TKI treatment, two patients were diagnosed with stage IIIB carcinoma and 60 with stage IV. The 62 patients included 34 with single and 28 with complex mutations. Twenty-nine patients carried an

Table I Clinicopathologic characteristics of patients with EGFR exon 20 mutations

\begin{tabular}{ll}
\hline Variable & Number \\
\hline Sex & \\
Male & 39 \\
Female & 23 \\
Age (years) & \\
Range & $30-76$ \\
Median & 60 \\
$\quad<65$ & 38 \\
$\geq 65$ & 24 \\
Smoking status & \\
Never & 31 \\
Former/current & 31 \\
PS & \\
0-I & 51 \\
2-3 & 11 \\
Histology & \\
Adenocarcinoma & 56 \\
Nonadenocarcinoma & 6 \\
Mutation type & \\
S768I & 10 \\
Insertion & 29 \\
T790M & 23 \\
EGFR-TKls & \\
First line & 3 \\
Second line & 24 \\
Further line & 35 \\
IIIB & \\
IV & \\
\hline
\end{tabular}

Abbreviations: EGFR, epidermal growth factor receptor; PS, performance status; TKI, tyrosine kinase inhibitor. 
Table 2 EGFR mutation patterns in 62 patients with exon 20 mutations

\begin{tabular}{ll}
\hline Mutation type & Number (\%) \\
\hline S768I & \\
Single & $3(30.00)$ \\
S768I + I9 delete & $3(30.0)$ \\
S768I + L858R & $4(40.0)$ \\
20 insertion & \\
Single & $26(89.7)$ \\
20 insertion + I9 delete & $2(6.9)$ \\
20 insertion + L858R & I $(3.4)$ \\
T790M & \\
Single & $5(21.7)$ \\
T790M + I9 delete & $6(26.1)$ \\
T790M + L858R & $10(43.5)$ \\
T790M + G7I9X & $1(4.3)$ \\
T790M + L86IQ & I (4.3) \\
\hline
\end{tabular}

Abbreviation: EGFR, epidermal growth factor receptor.

insertion, twenty-three showed T790M mutation, and ten carried S768I mutation (Table 2).

\section{Efficacy of exon 20 mutations}

The efficacy of EGFR-TKIs in patients was evaluated. The response data are shown in Table 3 . The patient response rate and disease control rate were $8.1 \%$ and $54.8 \%$, respectively. Among these, absence of CR, five cases of PR, and 29 cases of SD were confirmed. The median PFS and OS in all patients were 2.1 months and 13.3 months, respectively.

In the subset analysis, patients with S768I mutation had the most favorable PFS (median, 2.7 months), followed by those with T790M (median, 2.4 months) and insertion (median, 1.9 months) mutations $(P=0.022$; Figure $1 \mathrm{~A})$. However, no differences in OS were observed among the three groups (Figure 1B). The PFS of EGFR-TKIs treatment in eleven patients exceeded 6 months (Table 4).

Patients with single mutations had significantly shorter PFS than did patients with complex mutations ( 2.7 months vs 1.9 months, $P=0.034$; Figure $2 \mathrm{~A}$ ). No efficacy difference was found among single S768I, insertion, and T790M mutation patients ( 2.4 months vs 1.9 months vs 1.8 months, $P=0.457$ ). The OS of patients with single and complex mutations was not significantly different (Figure 2B).

\section{Efficacy comparison with common mutations}

In total, 742 patients treated with first-generation EGFRTKIs were selected among 1,560 patients harboring EGFR mutations. Among these 742 patients, 592 had deletion in exon 19 and L858R substitution in exon 21 (group A), 71 had exon 18 G719X and exon 21 L861Q (group B), 62 had exon 20 mutations (group C, current study data), and 17 had other mutations (group D). The PFS of groups A, B, C, and D with EGFR-TKIs treatment was 10.8 months, 6.2 months, 2.1 months, and 3.3 months, respectively $(P=0.015)$. The OS difference also varied among groups $\mathrm{A}$ and $\mathrm{B}$ and groups $\mathrm{C}$ and D (25.5 months vs 21.7 months, 13.3 months vs 16.5 months, respectively $P=0.041$ ).

\section{Discussion}

To the best of our knowledge, this is the first study that specifically demonstrated the clinical efficacy of EGFRTKIs in patients with exon 20 mutations as inferior to that of patients with common EGFR mutations. We also found that patients with exon 20 complex mutations have better PFS and OS than those carrying a single mutation.

The frequency of uncommon mutations ranged from $10 \%$ to $18 \%{ }^{18-21}$ The most common mutation was G719X in exon 18 , followed by L861Q in exon $21 .{ }^{13-15}$ These two mutations were moderately sensitive to first-generation EGFR-TKIs with a median PFS of $\sim 6$ months. Exon 20 mutations including insertions, S768I, and T790M are relatively rare compared with L861Q and G719X according to previous reports. ${ }^{16,17}$ In the present study, the frequency of exon 20 mutation was $5.7 \%$, which is consistent with previous studies.

The efficacy of EGFR-TKIs against exon 20 mutations is debatable. ${ }^{18,20,21}$ A preclinical study showed that the S768I mutant was resistant to EGFR-TKIs. ${ }^{22}$ By contrast, several small case studies demonstrated the clinical efficacy to EGFR-TKIs in patients carrying S768I mutations. ${ }^{23,24}$ In this study, ten patients were identified with S768I mutations with a clinical efficacy inferior to that of the other common mutations, but better than other exon 20 mutations (Figure 1).

Table 3 Efficacy of EGFR-TKIs in patients with EGFR exon 20 mutations

\begin{tabular}{lllllllll}
\hline Mutation type & CR & PR & SD & PD & ORR (\%) & DCR (\%) & PFS (months) & OS (months) \\
\hline S768I $(n=10)$ & 0 & 2 & 5 & 3 & 20 & 70 & 2.7 & 14.5 \\
20 insertion $(n=29)$ & 0 & 2 & 11 & 16 & 6.90 & 44.80 & 1.9 & 12.9 \\
T790M $(n=23)$ & 0 & 1 & 13 & 9 & 4.30 & 49.10 & 2.4 & 13.5 \\
\hline
\end{tabular}

Abbreviations: EGFR, epidermal growth factor receptor; TKI, tyrosine kinase inhibitor; CR, complete response; PR, partial response; SD, stable disease; PD, progressive disease; ORR, overall response rate; DCR, disease control rate; PFS, progression-free survival; OS, overall survival. 

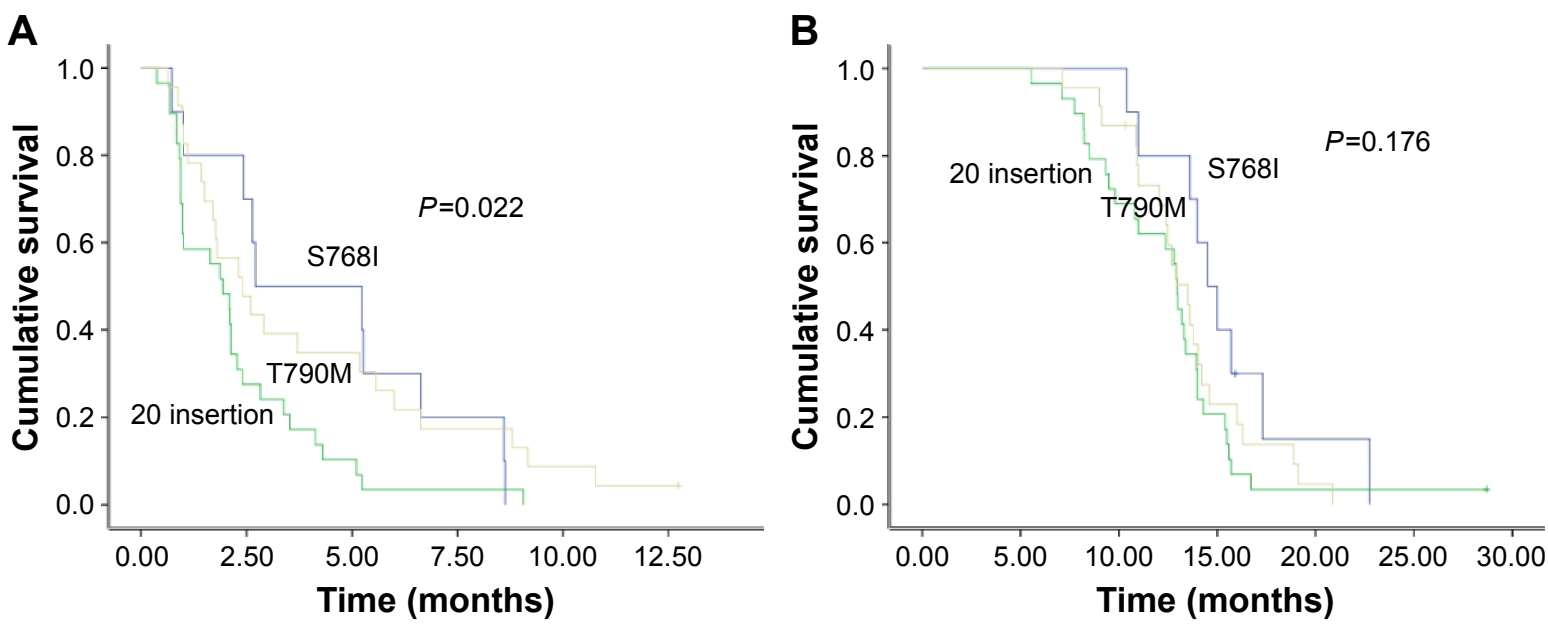

Figure I PFS and OS comparison in patients harboring different subtypes of EGFR exon 20 mutations.

Notes: (A) PFS and (B) OS in patients carrying T790M, S768I, and 20 insertion mutations.

Abbreviations: PFS, progression-free survival; OS, overall survival; EGFR, epidermal growth factor receptor.

Table 4 Clinical profile of patients with PFS of EGFR-TKIs treatment $>6$ months

\begin{tabular}{|c|c|c|c|c|c|c|c|}
\hline Case & Sex & $\begin{array}{l}\text { Age } \\
\text { (years) }\end{array}$ & Stage & Smoking & Gene type & $\begin{array}{l}\text { EGFR-TKIs } \\
\text { PFS (months) }\end{array}$ & $\begin{array}{l}\text { OS } \\
\text { (months) }\end{array}$ \\
\hline I & Female & 59 & IV & No & S768I & 8.6 & $67+$ \\
\hline 2 & Male & 66 & IIIB & No & T790M + 19 delete & 6.6 & 42 \\
\hline 3 & Male & 62 & IV & No & 20 insertion +19 delete & 8.7 & 34 \\
\hline 4 & Female & 56 & IV & No & T790M + L858R & 6.7 & $66+$ \\
\hline 5 & Male & 64 & IV & Yes & 20 insertion & 9.1 & $35+$ \\
\hline 6 & Female & 49 & IV & No & $S 768 I+L 858 R$ & 12.7 & 54 \\
\hline 7 & Male & 47 & IV & Yes & 20 insertion + L858R & 9.0 & $36+$ \\
\hline 8 & Male & 68 & IV & No & T790M + L858R & 10.7 & 55 \\
\hline 9 & Female & 63 & IV & No & T790M + G7I9X & 6.0 & $43+$ \\
\hline 10 & Female & 55 & IV & No & S768I + 19 delete & 8.8 & 25 \\
\hline II & Male & 57 & IV & Yes & 20 insertion & 8.0 & $46+$ \\
\hline
\end{tabular}

Abbreviations: PFS, progression-free survival; EGFR, epidermal growth factor receptor; TKI, tyrosine kinase inhibitor; OS, overall survival.
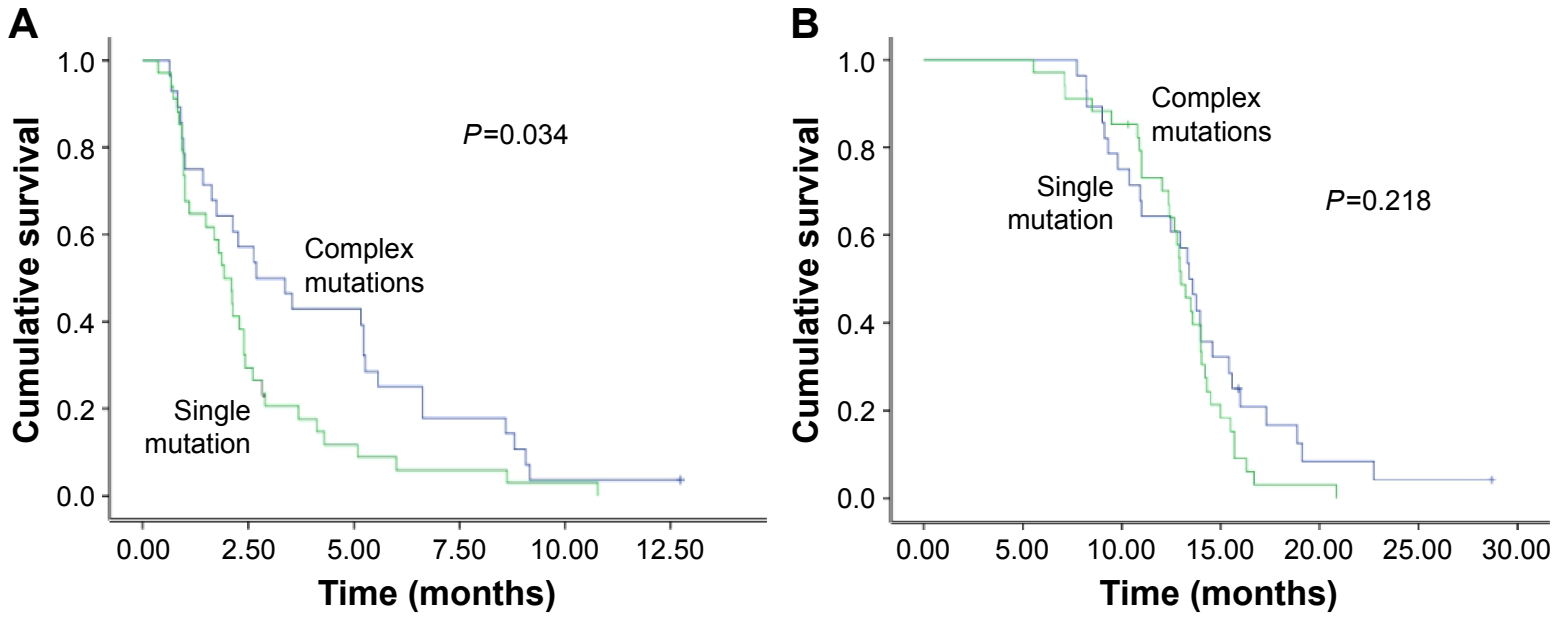

Figure 2 PFS and OS comparison between patients harboring EGFR exon 20 single mutations and complex mutations.

Notes: (A) PFS and (B) OS comparison in patients with single mutation and complex mutations.

Abbreviations: PFS, progression-free survival; OS, overall survival; EGFR, epidermal growth factor receptor. 
The PFS was 2.7 months with afatinib treatment in 23 patients harboring exon 20 insertion mutation in the study of Yang et al, ${ }^{25}$ suggesting that patients with this uncommon mutation may benefit from treatment with EGFR-TKIs. The PFS of exon 20 insertions was inferior to that in the study of Yang et al in the current cohort, which may be related to the first-generation EGFR-TKIs used.

In contrast to exon 20 insertions, most of the T790M mutations were concurrent with common mutations. Although considered as a common resistance mutation in patients who progressed from EGFR-TKIs treatment, only $\sim 1 \%-2 \%$ of patients harbored the de novo T790M mutation. ${ }^{19,25}$ Patients with this mutation responded to EGFR inhibitors, but with a shorter PFS than in patients with common mutations. ${ }^{25}$ In our cohort, 23 patients were confirmed with T790M de novo mutation, and the median PFS was 2.4 months. The results suggest that EGFR-TKIs benefited certain groups of patients. For patients with de novo T790M mutation, the emphasis on third-generation EGFR-TKIs targeting this mutation may be a better choice in future clinical practice.

In the present study, we found that the therapeutic efficacy against single mutations was inferior to that of complex mutations (Figure 2A). However, the efficacy difference of firstgeneration EGFR-TKIs for patients with single mutations did not exist. Eight of eleven patients with PFS $>6$ months harbored complex mutations in the current study (Table 4). We suggest that the treatment recommendations should focus on complex but not single mutations.

The current study is limited by the small size and a retrospective design.

The second-generation EGFR-TKIs demonstrated better efficacy than the first-generation products. ${ }^{25,26}$ Due to the nonapproval in the People's Republic of China, no data of patients treated with second-generation EGFR-TKIs were collected in the present study. The polymerase chain reactionbased amplification refractory mutation system method was used in the current study, which failed to cover all the insertion mutations in exon 20 and probably skewed the results.

\section{Conclusion}

Our results suggest that the efficacy of the first-generation EGFR-TKI treatment against the EGFR exon 20 mutations was inferior to treatments targeting the common mutations. The therapeutic efficacy in patients who harbored exon 20 mutations, concurrent with other common mutations, was better than in those carrying a single mutation. The efficacy of new drugs targeting the rare mutations needs to be elucidated in future studies.

\section{Disclosure}

The authors report no conflicts of interest in this work.

\section{References}

1. Chen WQ, Zheng RS, Zhang SW, Zeng HM, Zou XN. The incidences and mortalities of major cancers in China, 2010. Chin J Cancer. 2014; 33(8):402-405.

2. Chang JS, Chen LT, Shan YS, et al. Comprehensive analysis of the incidence and survival patterns of lung cancer by histologies, including rare subtypes, in the era of molecular medicine and targeted therapy: a nationwide cancer registry-based study from Taiwan. Medicine (Baltimore). 2015;94(24):e969.

3. Sun Y, Ren Y, Fang Z, et al. Lung adenocarcinoma from East Asian never-smokers is a disease largely defined by targetable oncogenic mutant kinases. J Clin Oncol. 2010;28(30):4616-4620.

4. Li H, Pan Y, Li Y, et al. Frequency of well-identified oncogenic driver mutations in lung adenocarcinoma of smokers varies with histological subtypes and graduated smoking dose. Lung Cancer. 2013;79(1):8-13.

5. Zhang Y, Sun Y, Pan Y, et al. Frequency of driver mutations in lung adenocarcinoma from female never-smokers varies with histologic subtypes and age at diagnosis. Clin Cancer Res. 2012;18(7):1947-1953.

6. Wu C, Zhao C, Yang Y, et al. High discrepancy of driver mutations in patients with NSCLC and synchronous multiple lung ground-glass nodules. J Thorac Oncol. 2015;10(5):778-783.

7. Zhou C, Wu YL, Chen G, et al. Erlotinib versus chemotherapy as first-line treatment for patients with advanced EGFR mutation-positive non-smallcell lung cancer (OPTIMAL, CTONG-0802): a multicentre, open-label, randomised, phase 3 study. Lancet Oncol. 2011;12(8):735-742.

8. Mitsudomi T, Morita S, Yatabe Y, et al; West Japan Oncology Group. Gefitinib versus cisplatin plus docetaxel in patients with non-small-cell lung cancer harbouring mutations of the epidermal growth factor receptor (WJTOG3405): an open label, randomised phase 3 trial. Lancet Oncol. 2010;11(2):121-128.

9. Mok TS, Wu YL, Thongprasert S, et al. Gefitinib or carboplatinpaclitaxel in pulmonary adenocarcinoma. NEngl J Med. 2009;361(10): 947-957.

10. Ciuleanu T, Brodowicz T, Zielinski C, et al. Maintenance pemetrexed plus best supportive care versus placebo plus best supportive care for non-small-cell lung cancer: a randomised, double-blind, phase 3 study. Lancet. 2009;374(9699):1432-1440.

11. Shi Y, Au JS, Thongprasert S, et al. A prospective, molecular epidemiology study of EGFR mutations in Asian patients with advanced non-small-cell lung cancer of adenocarcinoma histology (PIONEER). J Thorac Oncol. 2014;9(2):154-162.

12. Tanaka T, Matsuoka M, Sutani A, et al. Frequency of and variables associated with the EGFR mutation and its subtypes. Int J Cancer. 2010; 126(3):651-655.

13. Watanabe S, Minegishi Y, Yoshizawa H, et al. Effectiveness of gefitinib against non-small-cell lung cancer with the uncommon EGFR mutations G719X and L861Q. J Thorac Oncol. 2014;9(2):189-194.

14. Kancha RK, Peschel C, Duyster J. The epidermal growth factor receptorL861Q mutation increases kinase activity without leading to enhanced sensitivity toward epidermal growth factor receptor kinase inhibitors. J Thorac Oncol. 2011;6(2):387-392.

15. Chiu CH, Yang CT, Shih JY, et al. Epidermal growth factor receptor tyrosine kinase inhibitor treatment response in advanced lung adenocarcinomas with G719X/L861Q/S768I mutations. J Thorac Oncol. 2015;10(5):793-799.

16. Lee Y, Lee GK, Hwang JA, Yun T, Kim HT, Lee JS. Clinical likelihood of sporadic primary EGFR T790M mutation in EGFR-mutant lung cancer. Clin Lung Cancer. 2015;16(1):46-50.

17. Yasuda H, Park E, Yun CH, et al. Structural, biochemical, and clinical characterization of epidermal growth factor receptor (EGFR) exon 20 insertion mutations in lung cancer. Sci Transl Med. 2013;5(216): 216 ra 177. 
18. Pallan L, Taniere P, Koh P. Rare EGFR exon 20 S768I mutation predicts resistance to targeted therapy: a report of two cases. J Thorac Oncol. 2014;9(10):e75.

19. Beau-Faller M, Prim N, Ruppert AM, et al. Rare EGFR exon 18 and exon 20 mutations in non-small-cell lung cancer on 10117 patients: a multicentre observational study by the French ERMETIC-IFCT network. Ann Oncol. 2014;25(1):126-131.

20. Wu JY, Yu CJ, Chang YC, et al. Effectiveness of tyrosine kinase inhibitors on "uncommon" epidermal growth factor receptor mutations of unknown clinical significance in non-small cell lung cancer. Clin Cancer Res. 2011;17(11):3812-3821.

21. Yang JC, Shih JY, Su WC, et al. Afatinib for patients with lung adenocarcinoma and epidermal growth factor receptor mutations (LUX-Lung 2): a phase 2 trial. Lancet Oncol. 2012;13(5):539-548.

22. Chen YR, Fu YN, Lin CH, et al. Distinctive activation patterns in constitutively active and gefitinib-sensitive EGFR mutants. Oncogene. 2006;25(8):1205-1215.
23. Hellmann MD, Reva B, Yu H, et al. Clinical and in vivo evidence that EGFR S768I mutant lung adenocarcinomas are sensitive to erlotinib. J Thorac Oncol. 2014;9(10):e73-e74

24. Masago K, Fujita S, Irisa K, et al. Good clinical response to gefitinib in a non-small cell lung cancer patient harboring a rare somatic epidermal growth factor gene point mutation; codon 768 AGC $>$ ATC in exon 20 (S768I). Jpn J Clin Oncol. 2010;40(11):1105-1109.

25. Yang JC, Sequist LV, Geater SL, et al. Clinical activity of afatinib in patients with advanced non-small-cell lung cancer harbouring uncommon EGFR mutations: a combined post-hoc analysis of LUXLung 2, LUX-Lung 3, and LUX-Lung 6. Lancet Oncol. 2015;16(7): $830-838$.

26. Heigener DF, Schumann C, Sebastian M, et al; Afatinib Compassionate Use Consortium (ACUC). Afatinib in non-small cell lung cancer harboring uncommon EGFR mutations pretreated with reversible EGFR inhibitors. Oncologist. 2015;20(10):1167-1174.
OncoTargets and Therapy

\section{Publish your work in this journal}

OncoTargets and Therapy is an international, peer-reviewed, open access journal focusing on the pathological basis of all cancers, potential targets for therapy and treatment protocols employed to improve the management of cancer patients. The journal also focuses on the impact of management programs and new therapeutic agents and protocols on

\section{Dovepress}

patient perspectives such as quality of life, adherence and satisfaction. The manuscript management system is completely online and includes a very quick and fair peer-review system, which is all easy to use. Visit http://www.dovepress.com/testimonials.php to read real quotes from published authors. 